\title{
Proactive Engineering and PLM: Current Status and Research Challenges
}

\author{
Frédéric Demoly ${ }^{1, \star}$, Henk Jan Pels ${ }^{2}$, and Samuel Gomes ${ }^{1}$ \\ 1 IRTES-M3M, UTBM, 90010 Belfort Cedex, France \\ \{frederic.demoly, samuel.gomes\}@utbm.fr \\ 2 Technische Universiteit Eindhoven, Eindhoven, The Netherlands \\ h.j.pels@tue.nl
}

\begin{abstract}
This paper discusses about the evolution of Product Lifecycle Management (PLM) through the introduction of an emerging vision in engineering design, proactive engineering. Over the last two decades, engineering design has seen some relevant approaches covering sequential engineering and then concurrent engineering $(\mathrm{CE})$. Indeed, this shift was required to encompass knowledge integration issue into product design stages. This has led to relevant approaches such as design for X, parametric design, PLM-based approaches, decision-making support and ontology-based approaches to name a few. Proactive engineering can be considered as an emerging engineering framework which integrates as early as possible lifecycle knowledge and technological constraints in product design and manage those knowledge in an integrated and harmonious manner. The fact of using lifecycle process knowledge as design context demands therefore the definition of downstream processes before defining he product geometry so as to overcome current limitations in CE oriented PLM approaches. Hence, with such stakes, understanding and awareness becomes crucial in PLM in order to deliver well-balanced products.
\end{abstract}

Keywords: Product Lifecycle Management; Proactive engineering, Qualitative description, Understanding, Awareness in design.

\section{Introduction}

Nowadays companies need to be more competitive (externally focused) and productive and efficient (internally focused) to deliver personalizable products which are lifecycle-friendly oriented [1]. Over the last decades, engineering design has incrementally shifted to different engineering paradigms in order to overcome industrial stakes and research challenges, especially the optimisation of product development lead time and knowledge integration in product design [2 4]. This has led to successful methods enabling the promotion of Concurrent Engineering (CE) philosophy and Product Lifecycle Management (PLM) vision [5]. Nevertheless, product design still required a more efficient verification and validation

\footnotetext{
* Corresponding author.
} 
procedure regarding lifecycle stages expectations [6], and suitable and anticipatory contexts for designers based on process procedural knowledge [7, 8]. Currently, the concurrent consideration of lifecycle constraints and knowledge does not provide enough awareness and understanding for stakeholders, especially for designers [1]. The traditional "pass the torch" (in other words: "over the wall" issue) exchange procedure over lifecycle phases and involved actors is still a critical step with some barriers [9]. This can be understood as the lack of rationale, intents, logic on product design decisions and its impact on downstream processes, and the need of appropriate product representations in line with stakeholders' viewpoints and concerns [10]. This statement can be confirmed regarding the current concurrent product design process as well as the associated PLM processes. The proposed work does not describe a comprehensive state-of-the-art review in engineering design and PLM - as successfully realised in [3, 5] -, but particularly addresses its current limitations to fully integrated lifecycle phases and related constraints and knowledge in product design. Hence this paper discusses the required evolution of PLM - as initiated in [11] - through the introduction of proactive engineering as an emerging paradigm in engineering design. Over the last two decades, engineering design has seen some relevant approaches covering sequential engineering and then CE [12]. Indeed, this shift was required to encompass the knowledge integration issue into product design stages. This has led to relevant approaches such as design for $\mathrm{X}$ (DFX) [13], parametric design, PLM-based approaches, decision-making support and ontology-based approaches [14] to name a few. Proactive engineering can be considered as an emerging engineering framework which integrates as early as possible lifecycle knowledge - described in a formal manner for example - and technological constraints in product design and manage this knowledge in an integrated and harmonious manner [15, 1, 16]. The fact of using lifecycle process knowledge in design context [7] demands therefore the definition of downstream processes (i.e. lifecycle sequence planning) before defining he product detailed geometry, so as to overcome current limitations in CE oriented PLM approaches. Therefore with such stakes, understanding and awareness aspects become crucial in PLM in order to deliver well-balanced products (i.e. products fulfilling lifecycle constraints and knowledge in a harmonious and consistent manner), while considering $\mathrm{X}$ planning definition where $\mathrm{X}$ stands for manufacturing, assembly, disassembly, maintenance, transport, etc. In such a way, traditional DFX approaches need to be turned back towards "X sequence planning for design".

Following this original point of view, the paper highlights in Section 2 the current stakes in the CE paradigm with the recent birth of proactive engineering. Then, Section 3 presents the remaining challenges and emerging needs in PLM regarding this novel paradigm. Finally, Section 4 addresses the description of a proactive design framework within PLM. The final outcome of this proposal is to propose a novel shift in both product design and PLM, and give new directions for further research activities in these fields. 


\section{Current Stakes in Concurrent Engineering}

At a critical place, the product design phase requires the consideration and integration of all constraints and knowledge (business processes, business terms, expert rules, job experience, etc.) of product lifecycle phases. This implies to embrace a large amount of rules which increases the work complexity of product architects and designers [6], and consequently increases computational complexity of the design phase [10]. Over the past two decades, this issue has been tackled by shifting from sequential engineering to $\mathrm{CE}$, therefore facilitating the integration of specific concerns (i.e. manufacturing knowledge, assembly knowledge, etc.) into product detailed design stages with the support of expert systems and inference engines [12]. It can be noticed that this shift has generated potential gains by using heuristics rules with associated quantitative engineering data 17, 18]. Thus a set of relevant design for X (DFX) and design to X (DTX) components were proposed [12, 19]. These approaches can be understood in a way that DFX components gather numerous rules and constraints and DTX components concern properties values to be checked 20]. Literature has provided numerous published research works in the above-listed fields, among them Design For Assembly (DFA) seems to be the most investigated component in DFX. These approaches can be considered as semi-generative and based on heuristics and geometrics rules in order to tackle current difficulties in the management of the product structure complexity and related product modelling [17]. Recent research efforts in proactive DFA (also called assembly oriented design) have proven that the early generation of admissible assembly sequences during conceptual design stages can be created in order to provide an appropriate contextual support for assembly design and modelling phases [17, 18], even for the geometric definition in a top-down manner 21]. Other DFX approaches are still described while using assessment techniques in order to evaluate the current product design according to specific rules and constraints [22]. Relevant future trends in decision-based DFX can also be found in [13]. Moreover, the combinatorial complexity of processing knowledge and rules and the need of agility in design has been partially covered by knowledge-based engineering (KBE) techniques and applications in detailed design stage [23].

Today, the CE philosophy has reached its limit or at least has some pending issues, and some key points can be introduced to argue this statement. As such, the current CE-based approaches do not provide enough reasoning layer for a full understanding and awareness of product architects and designers. Indeed, designers need to reason and understand the context in which the design is carried out, that is why a qualitative description layer would be an added value 24]. Knowledge from lifecycle processes is not yet considered as design aid but as verification and validation procedure in the detailed design stage [6]. This fact demonstrates that lifecycle knowledge has to be considered in a way that aids designers from preliminary design stage, therefore improving designer's awareness and anticipation 25]. In such a way, it is important to address a proactive vision of the product development by considering downstream processes as early as possible in product design. Thus awareness and understanding will be promoted 
to product/process architects and designers. This can be done with relevant data input and some specific layers which may introduce qualitative context based on formal description. Here qualitative context may include formal description of engineering intents and knowledge such as explained in 24]. Another relevant aspect is the way of knowledge reuse, if lifecycle context in design is required, it therefore demands an appropriate injection process of knowledge. An interesting research effort towards knowledge on-demand procedures based on context capturing would provide benefits in design phase [7, 3, 27].

As a synthesis, current stakes in engineering can be represented in Figure 1. where engineering shifts from sequential to $\mathrm{CE}$ and $\mathrm{CE}$ to proactive engineering are shown. In the past, sequential engineering meant the execution of engineering tasks without overlapping and information exchange were made possible with quantitative data. Based on this process, CE has provided gains in BeginningOf-Life (BOL) by overlapping detailed design stage with manufacturing planning and then assembly planning, etc. This was enabled with the support of heuristic rules and parameters to be considered in design. More recently, proactive engineering aims to fully overlap engineering phases based on minimum design information, on which manufacturing and assembly planning can be generated and considered to define a qualitative context for designers as early as possible in the preliminary design stage. As result, with such emerging paradigm, product development lead time becomes more optimised and more efficient by improving understanding and awareness of designers decisions, and promoting knowledge activation and injection in design in an appropriate manner.

\section{Remaining Challenges and Emerging Needs in PLM}

If proactive engineering is designing products for optimal performance over its lifecycle, then it needs a consistent PLM strategy since PLM is about managing knowledge on the product and all its lifecycle processes in order to optimise product lifecycle benefits and minimise product lifecycle cost [28, 29]. In the PLM context a suitable definition of knowledge is that of [30, 31] : K = I .E.S.A, where K stands for Knowledge, I for Information, $\mathrm{S}$ for Skill, E for Experience and $\mathrm{A}$ for Attitude. This definition takes the pragmatical view that knowledge is what enables a person (or a machine) to perform a task. If two different persons execute the same task with different performance in terms of cost, time and quality, than the difference is explained by different knowledge. To perform a task a certain skill is needed. Skill is obtained by teaching (from documents or by imitation). The more often the task is repeated, the more the performance increases. This is the Experience factor. In most cases the execution of a task requires some choices or decisions to be taken. More information enables better decisions and thus better task performance. Finally, the person may have skill, experience and information; if he is not motivated there will be little or no result.

The product lifecycle encompasses many different processes that require many tasks to be executed like design, manufacturing, assembly, installation, operation, maintenance, refurbishing and demolition. Product lifecycle performance 


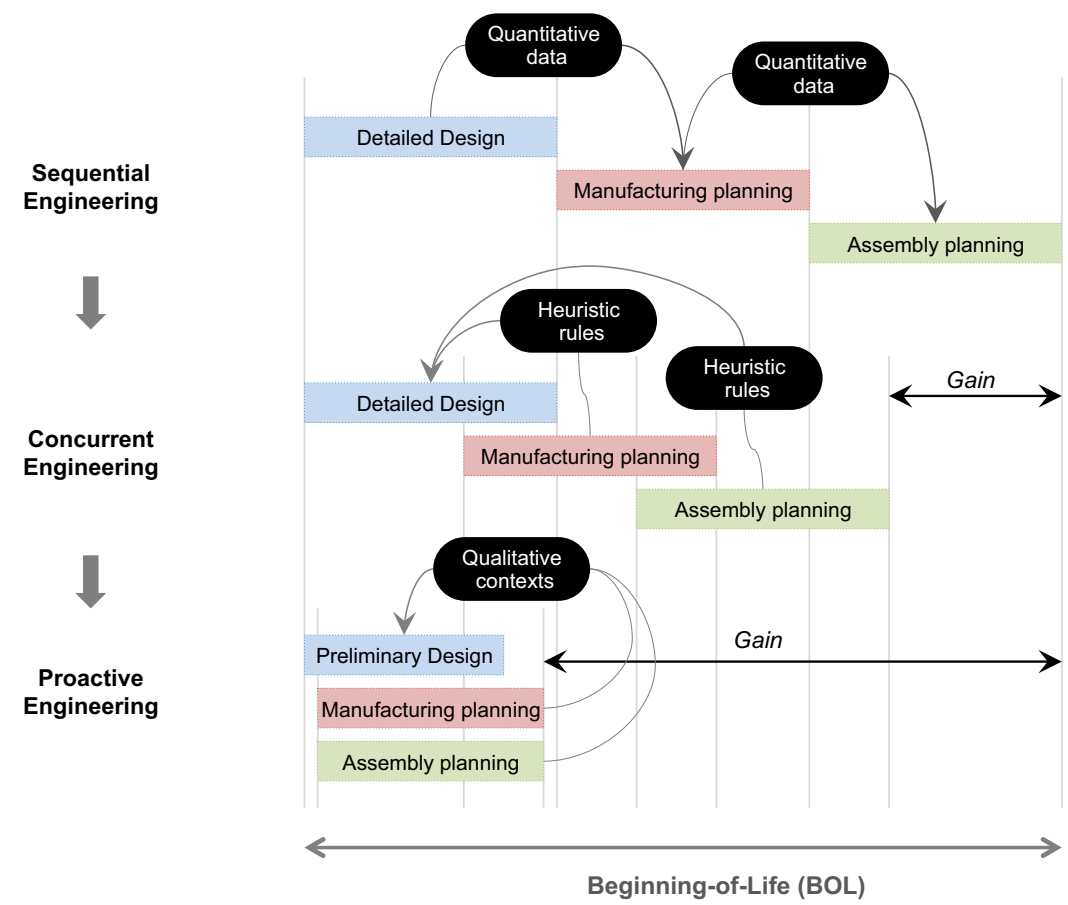

Fig. 1. Towards a new engineering shift: proactive engineering

depends by definition on the knowledge applied in all those tasks. Like the core function of ERP (Enterprise Resource Planning) is to make all resources available to perform a particular tasks when and where it is to be executed, the core function of PLM is make the proper knowledge available in each resource. Product development creates and documents the knowledge. Human resources organizes the hiring and training to acquire skill and should record the experience. Information systems organize the availability of information and management takes care for Attitude. Knowledge is increasingly dynamic so a main competitive capability (internally focused) is to get new knowledge faster in operation in all lifecycle processes.

Three flows of knowledge can be distinguished. The first is from new technology to be implemented in reliable product design. The second is from new customer needs to be implemented in a product serving that customer. The third is from new knowledge on possible process improvement from the field into new knowledge available in the resources executing those processes. This third one is the object of proactive engineering, i.e. observe product behaviour in different lifecycle processes, generate ideas for improvements (or sequence planning) and implement those ideas (i.e. as contextual information) in new product and process designs. Currently in most cases the flow of knowledge from real life processes to product development is very slow and narrow. An important reason is that by the time the downstream processes like maintenance are starting, 
product development is already working on the next product generation. When knowledge flows faster along the three axes mentioned above, this problem will get smaller.

PLM has basically three ways of improving product development performance: 1) eliminate non value adding activities from the process to reduce cost, 2) reduce waiting times in knowledge flows in order to reduce time to operation and 3) stimulate reuse of knowledge in order to increase quality, efficiency and anticipation. The last one is the most powerful to improve product lifecycle value. Reuse in design is achieved first by reusing existing components and modules, but second by parameterizing the design (via rules and parameters). The effect is that the knowledge for a class of specific product types is abstracted to the level of a single product family via a consistent configuration of rules and parameters. Then product and process specifications for specific products can be derived cheap, fast and reliable - by feeding parameter values into the product family models.

In the context of proactive engineering product families are important because family specifications have a longer lifetime, change slower, that those of individual products. This means that experience with process families can be collected and used while the product family is still alive, thus enabling faster learning of process designers. Another important PLM feature for proactive design is enabling concurrent engineering, meaning that downstream processes are designed concurrently with the product. Both the new product and the new process are to be designed as a limited set of changes to and existing product and its processes. Most component (families) are to be reused without change, while only specific components and processes may be redesigned. Thus the knowledge in the stable part can be reused, has a longer life, more repetitive application so the knowledge can be of much better quality. Not only for the resource applying the knowledge repetition is important to create experience, also for the knowledge creation process it holds that to each repetition of application gives a possibility to learn and improve the specification of the skill.

As a result, current PLM vision requires evolution towards the effective and consistent management of knowledge [11], the improvement of knowledge reuse from downstream processes in product design and the appropriate knowledge representation and reasoning 32]. Among knowledge types, PLM still requires the consideration and the qualitative and formal description of design intents with declarative knowledge (know-what), rationale with causal knowledge (Know-Why), process intents with procedural knowledge (Know-How) and temporal knowledge (KnowWhen) so as to increase awareness and understanding of information and knowledge flows 33]. Computational layers should also be introduced in order to facilitate information and knowledge processing between each engineering phases. In this context, mediator-based applications [34], hub-based applications, contextaware and context-sensitive applications, and knowledge on-demand mechanisms [35] with the support of reasoning features and agents [36] would provide an interesting "peacemaker" for existing PLM ecosystems. The peacemaker concept here will ensure the closed-loop of information and knowledge flows [37] and promotion 
of information irrigation with semantic federation [38] for various abstraction levels of information [39].

Figure 2 presents a summary of the main existing PLM systems (grey boxes) and applications (white boxes) covering the product lifecycle and associated to specific content (i.e. what, how, when and where). This map is composed of PLM systems which manages spatial and temporal information related to the product and its lifecycle processes (i.e. Product Data Management - PDM, Manufacturing Process Management - MPM and ERP), and applications which defines and optimizes product and process definitions. Hub and bridge applications have not been represented since these applications remains too specific regarding current research efforts. Here "what" is about technical entities and engineering data related to the product, "how" is about processes and functions, "when" addresses temporal events, and "where" denotes places and networks such as initiated in the Zachman's framework [40]. The "why" part (i.e. rationale and motivation) is not addressed yet, or at least not commonly agreed yet by the PLM community. This figure also highlights some overlaps along the lifecycle phases axis and some gaps about the content orientation axis. A critical feature is the lack of connection between content types, where continuity, logic and associated reasoning mechanisms of information and knowledge flows are currently missing in PLM ecosystems, especially in BOL phase. Indeed, current information exchange procedures are not sufficient to understand stakeholders' intents. Hence there is an opportunity to improve current position of PLM systems in order to promote proactive engineering by introducing new reasoning and computing layers on semantics and logics aspects in a central manner.

\section{Towards a Proactive Design Framework within PLM}

Proactive design implies that not only knowledge about the product technology, but knowledge about all lifecycle processes is used in optimizing the design of not only the product, but also that of all lifecycle processes. It means that each designers in the product lifecycle must have access to knowledge from all other designers. In such a way, The proposed proactive design framework, presented in Figure 3, introduces some new knowledge processing mechanisms in order to link "what" with "how" and "when" contents of PLM in a way that considers "X sequence planning for design" (DFX is actually turned back). The breakthrough lies in the understanding of design and process contexts and related designer's intents, and the description and instantiation of knowledge on-demand for its appropriate injection (i.e. accurate reuse) in the design process. As a result, the proposed framework, which provide a novel "why" layer for processing and controlling information and knowledge flows between product design and lifecycle sequence planning, can be deployed as follows:

Step 1. Generation of lifecycle (manufacturing, assembly, etc.) sequence planning. Based on preliminary product design input (e.g. bill of material, partto-part relationships, etc.), it is then possible to generate admissible lifecycle 


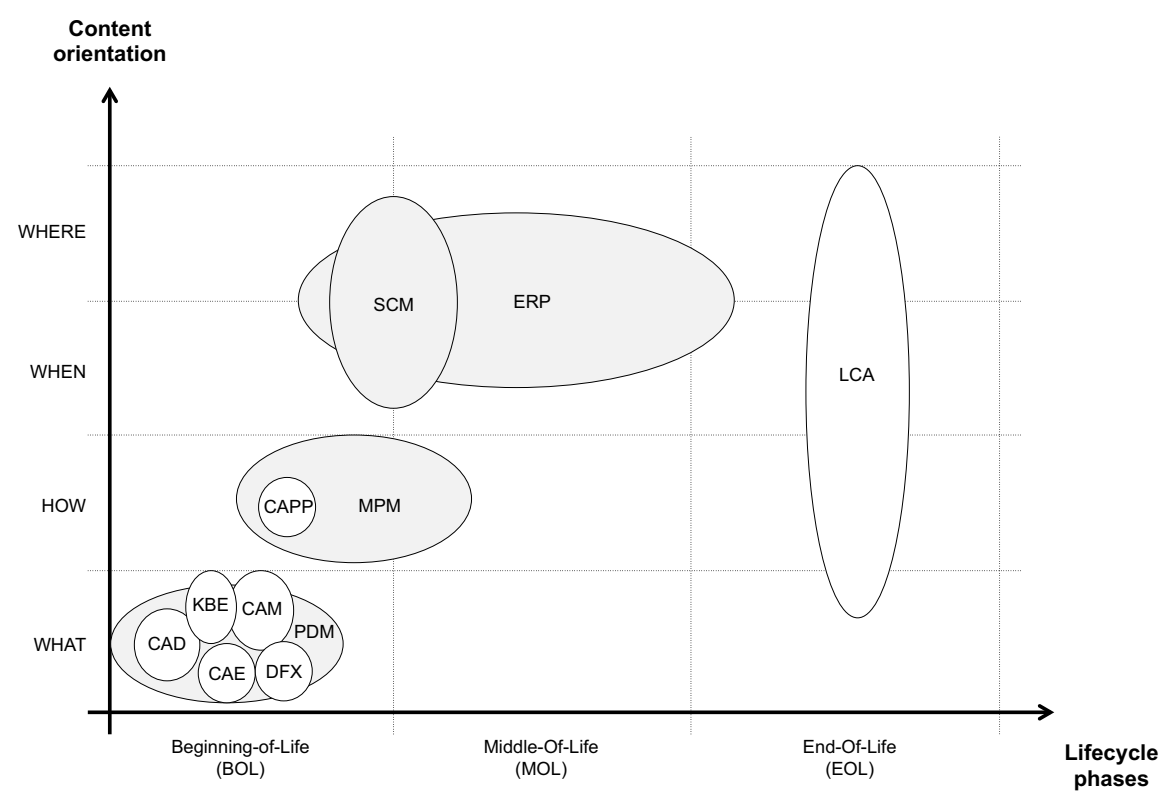

Fig. 2. Map of existing PLM systems and applications along lifecycle phases and content orientation

sequence planning (e.g. assembly sequence planning), on which a good one can be identified based on process planner experience [17, 18].

Step 2. Product structuring. The early-defined assembly sequence for example can be consistently considered in product design for product structuring. The same reasoning procedure can be done via the definition of the manufacturing sequence for part features structuring.

Step 3 \& 3'. Knowledge on-demand in design. This action is required and active all along the design process, from conceptual to detailed design stages. At any time, it should be possible to interpret the designer's context (in CAD modelling for example) in a way to understand and capture its design intents with surroundings engineering data related to the product. Here the captured intents are then described with declarative knowledge (knowing what).

Step 4 \& 4'. Knowledge on-demand in process planning. Similar to the previous step, the interpretation of process context is also needed at any time in order to describe planner's intents with procedural knowledge (knowing how) and temporal knowledge (knowing when).

Step 5. Knowledge consistency checking. This step links declarative knowledge with procedural and temporal knowledge by introducing causal knowledge (i.e. rationale). In such a way, knowledge about "what" and "how" can be described and linked so as to check knowledge consistency with logical inferences. 


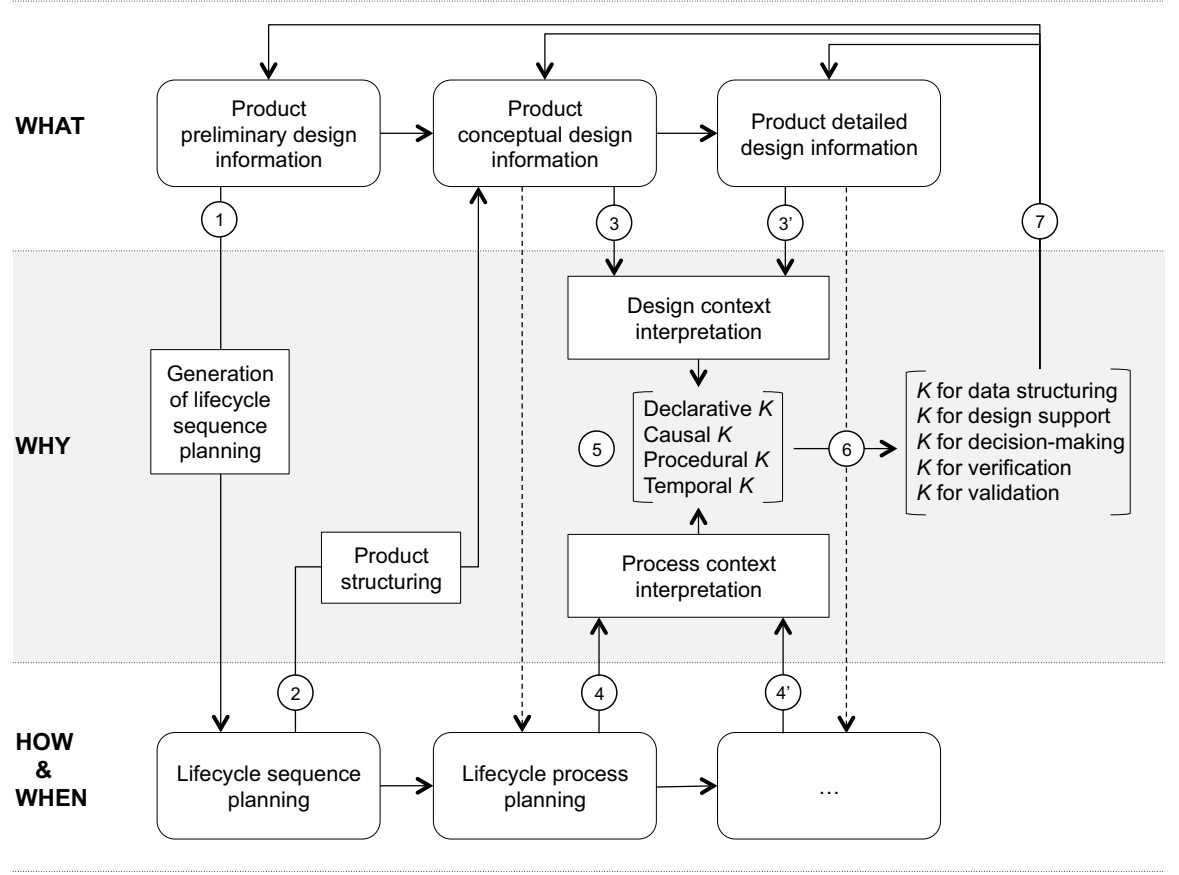

Fig. 3. Proactive design framework ( $K$ stands for Knowledge)

Step 6. Knowledge activation and instantiation. Based on the previous descriptive knowledge and the captured contexts, the needed knowledge is activated and instantiated towards the context purpose (i.e. data structuring, design support, decision-making, verification and validation).

Step 7. Knowledge injection in design. Finally, once activated, the knowledge is injected in the appropriate format (i.e. qualitative description) to the right person at the right place in the design process.

\section{Conclusions and Future Works}

This paper has highlighted current stakes and limits in concurrent engineering towards the need of a proactive engineering vision in consistency with PLM emerging needs. The authors have presented how traditional approaches can be turned back (e.g. DFX methods) by considering lifecycle sequence planning for design so as to promote awareness and understanding in design. Built on this, a novel proactive design framework within PLM has been proposed in order to link "what" with "how" and "when" contents by introducing an emerging "why" layer, where contexts and intents are described with qualitative knowledge (i.e. declarative, procedural , temporal and causal knowledge). This framework provides knowledge on-demand mechanisms and some consistency checking procedures in order to facilitate the knowledge injection process in product design. 
Hence future works will address each step of the proposed framework with specific mechanisms and algorithms. In addition, a PLM implementation will be conducted in order to introduce the missing "why" layer for linking existing PLM components (information systems and applications) in BOL phase.

\section{References}

1. Demoly, F., Deniaud, S., Gomes, S.: Towards an harmonious and integrated management approach for lifecycle planning. In: Emmanouilidis, C., Taisch, M., Kiritsis, D. (eds.) APMS 2013, Part II. IFIP AICT, vol. 398, pp. 208-215. Springer, Heidelberg (2013)

2. Sapuan, S.M., Osman, M.R., Nukman, Y.: State of the art of the concurrent engineering technique in the automotive industry. Journal of Engineering Design 17(2), 143-157 (2006)

3. Liu, S., Boyle, I.M.: Engineering design: perspectives, challenges, and recent advances. Journal of Engineering Design 20(1), 7-19 (2009)

4. Demoly, F., Yan, X.-T., Eynard, B., Gomes, S., Kiritsis, D.: Integrated product relationships management: a model to enable concurrent product design and assembly sequence planning. Journal of Engineering Design 23(7), 544-561 (2012)

5. Liu, W., Zeng, Y., Matlez, M., Brisson, D.: Product lifecycle management: a review. In: ASME International Design Engineering Technical Conferences (IDETC) and Computers and Information in Engineering Conference, DETC 2009-86983, San Diego, August 30-September 2 (2009)

6. Maropoulos, P.G., Ceglarek, D.: Design verification and validation in product lifecycle. CIRP Annals - Manufacturing Technology 59(2), 740-759 (2010)

7. Hong, J., Suh, E.-H., Kim, J., Kim, S.: Context-aware system for proactive personalized service based on context history. Expert Systems with Applications 36, 7448-7457 (2009)

8. Song, B., Jiang, Z.: Proactive search enabled context-sensitive knowledge supply situated in computer-aided engineering. Advanced Engineering Informatics 27, 6675 (2013)

9. Derrick, J., Wehrheim, H.: Model transformations across views. Science of Computer Programming 75, 192-210 (2010)

10. Kusiak, A., Salustri, F.A.: Computational intelligence in product design engineering: review and trends. Transactions on Systems, Man, and Cybernetics 37(5), 766-778 (2007)

11. Ameri, F., Dutta, D.: Product Lifecycle Management: Closing the Knowledge Loops. Computer-aided Design \& Applications 2(5), 577-590 (2005)

12. Huang, G.Q., Lee, S.W., Mak, K.L.: Web-based product and process data modeling in concurrent "design for X". Robotics and Computer-Integrated Manufacturing 15, 53-63 (1999)

13. Holt, R., Barnes, C.: Towards an integrated approach to "Design for X": an agenda for decision-based DFX research. Research in Engineering Design 21, 123-136 (2010)

14. Matsokis, A., Kiritsis, D.: An ontology-based approach for Product Lifecycle Management. Computers in Industry 61(8), 787-797 (2010)

15. Dencker, K., Fasth, A., Stahre, J., Martensson, L., Lundholm, T., Akillioglu, H.: Proactive assembly systems-realising the potential of human collaboration with automation. Annual Reviews in Control 33, 230-237 (2009) 
16. Wu, J.-W., Tseng, J.C.R., Yu, W.-D., Yang, J.-B., Lee, S.-M., Tsai, W.-N.: An integrated proactive knowledge model for enhancing engineering services. Automation in Construction 24, 81-88 (2012)

17. Demoly, F., Yan, X.-T., Eynard, B., Rivest, L., Gomes, S.: An Assembly oriented design framework for product structure engineering and assembly sequence planning. Robotics and Computer-Integrated Manufacturing 27(1), 33-46 (2011)

18. Demoly, F., Troussier, N., Eynard, B., Falgarone, H., Fricero, B., Gomes, S.: Proactive assembly oriented design approach based on the deployment of functional requirements. Journal of Computing Information and Science in Engineering 11(1), 014501-1 (2011)

19. Kuo, T.C., Huang, S.H.H., Zhang, H.C.: Design for manufacture and design for $\mathrm{X}$ : concepts, applications, and perspectives. Computers \& Industrial Engineering 41(3), 241-260 (2001)

20. Kimura, F., Matoba, Y., Mitsui, K.: Designing Product Reliability based on Total Product Lifecycle Modelling. CIRP Annals - Manufacturing Technology 56(1), 163$166(2007)$

21. Demoly, F., Toussaint, L., Eynard, B., Kiritsis, D., Gomes, S.: Geometric skeleton computation enabling concurrent product engineering and assembly sequence planning. Computer-Aided Design 43(12), 1654-1673 (2011)

22. Lambert, A.J.D., Gupta, S.M.: Disassembly modeling for assembly, maintenance, reuse and recycling (resource management). CRC Press (2004) ISBN-10: 1574443348

23. Myung, S., Han, S.: Knowledge-based parametric design of mechanical products based on configuration design method. Expert Systems with Applications 21(2), 99-107 (2001)

24. Demoly, F., Matsokis, A., Kiritsis, D.: A mereotopological product relationship description approach for assembly oriented design. Robotics and ComputerIntegrated Manufacturing 28(6), 681-693 (2012)

25. Zamenopoulos, T., Alexiou, K.: Towards an anticipatory view of design. Design Studies 28, 411-436 (2007)

26. Liu, Z., Xiao, J., Huang, Y.: Proactive product quality control: An integrated product and process control approach to MIMO systems. Chemical Engineering Journal 149, 435-446 (2009)

27. Nevo, D., Benbasat, I., Wand, Y.: The knowledge demands of expertise seekers in two different contexts: knowledge allocation versus knowledge retrieval. Decision Support Systems 53(3), 482-489 (2012)

28. Stark, J., et al.: Product Lifecycle Management - Paradigm for 21st century. Springer, Berlin (2004)

29. Terzi, S., Bouras, A., Dutta, D., Garetti, M., Kiritsis, D.: Product lifecycle management - from its history to its new role. International Journal of Product Lifecycle Management 4(4), 360-389 (2010)

30. Song, X.M., van der Bij, J.D., Weggeman, M.C.D.P.: Determinants of the level of knowledge application: a knowledge-based and information-processing perspective. Journal of Product Innovation Management 22(5), 430-444 (2005)

31. Song, X.M., van der Bij, J.D., Weggeman, M.C.D.P.: Factors for improving the level of knowledge generation in new product development. R\&D Management 36(2), 173-187 (2006)

32. Chandrasegaran, S.K., Ramani, K., Sriram, R.D., Horvath, I., Bernard, A., Harik, R.F., Gao, W.: The evolution, challenges, and future of knowledge representation in product design systems. Computer-Aided Design 45(2), 204-228 (2013) 
33. Kim, K.-Y., Kim, Y.S.: Causal design knowledge: Alternative representation method for product development knowledge management. Computer-Aided Design 43(9), 1137-1153 (2011)

34. Madhusudan, T.: An Intelligent Mediator-Based Framework for Enterprise Application Integration. Journal of Computing and Information Science in Engineering 4, 294-304 (2005)

35. Lee, H.J., Kim, J.H.: Mining knowledge demands from information flow. Expert Systems with Applications 39(8), 6799-6806 (2012)

36. Fan, X., Yen, J., Volz, R.A.: A theoretical framework on proactive information exchange in agent teamwork. Artificial Intelligence 169, 23-97 (2005)

37. Jun, H.-B., Kiritsis, D., Xirouchakis, P.: Research issues on closed-loop PLM. Computers in Industry 58, 855-868 (2007)

38. Fabian, B., Kunz, S., Konnegen, M., Müller, S., Günther, O.: Access control for semantic data federations in industrial product-lifecycle management. Computers in Industry 63, 930-940 (2012)

39. Helms, R.W.: Product Data Management as enabler for Concurrent Engineering. Thesis (PhD). Eindhoven University of Technology, The Netherlands (2002)

40. Zachman, J.A.: A Framework for Information Systems Architecture. IBM Systems Journal 26(3), 276-292 (1987)

41. Feng, G., Cui, D., Wang, C., Yu, J.: Integrated data management in complex product collaborative design. Computers in Industry 60, 48-63 (2009) 